\title{
MÚLTIPLAS FUNÇÕES DOS ARBORETOS DO PARQUE ESTADUAL DE PORTO FERREIRA, SP.
}

\author{
Sonia Aparecida de Souza Evangelista ${ }^{1}$ \\ Ernesto Pedro Dickfeldt ${ }^{2}$ \\ Suélen Rigon Bento da Fonseca ${ }^{3}$
}

\section{RESUMO}

Ao longo dos anos, três arboretos foram implantados no Parque Estadual de Porto Ferreira de forma multidisciplinar e participativa, integrando os programas de manejo da unidade. As coleções juntas somam 94 espécies que favoreceu a recuperação de áreas degradadas, o desenvolvimento de atividades de educação e interpretação ambiental, a conservação da biodiversidade, além de serem espaços de vivência e contemplação da natureza, desempenhando assim funções ambientais, educativas e sociais.

PALAVRAS-CHAVE: Recuperação. Educação Ambiental. Biodiversidade.

\section{INTRODUÇÃO}

Os arboretos são definidos como coleções vivas de árvores, arbustos, plantas medicinais, ornamentais e outras, mantidas cientificamente, documentadas e identificadas com finalidade de educação, pesquisa científica, material didático e fornecimento de sementes (BAZARETTI et al., 2010).

Nos parques e outras áreas protegidas a implantação de arboretos tem finalidade técnica, científica e educativa, sendo formados por uma única espécie ou por diversas.

No Parque Estadual Alberto Löfgren nove arboretos foram implantados ao longo dos anos, destacando-se o da Vila Amália e o Arboreto 500 Anos. Essas coleções são utilizadas como estratégias do programa de uso público em atividades

\footnotetext{
${ }^{1}$ Parque Estadual de Porto Ferreira - IF/SMA soniasouza@if.sp.gov.br

2 Parque Estadual de Porto Ferreira - IF/SMA ernesto.dickfeldt@terra.com.br

${ }^{3}$ Parque Estadual de Porto Ferreira - BK Consultoria Ambiental srigon5@yahoo.com.br
} 
interpretativas, educativas e contemplativas por visitantes de diferentes perfis (HERCULIANI et al., 2009), além da pesquisa científica.

A formação do primeiro arboreto no PEPF data de 1995 quando iniciou a implantação de instalações do programa de uso público. Atualmente esse programa atende a comunidade escolar, em geral e turistas, desenvolvendo atividades educativas, interpretativas, vivências e contato com a natureza.

Nesse trabalho, apresentamos o relato da implantação de três arboretos ao longo dos últimos 20 anos integrando atividades dos programas de manejo da unidade de conservação.

\section{OBJETIVOS}

- promover a recuperação das áreas degradadas no interior do PEPF,

- formar coleções vivas de espécies representativas dos ecossistemas locais e regionais,

- propiciar aos visitantes conhecimentos, contato e vivências com a flora local e regional, e

- integrar atividades dos programas de pesquisa, manejo do meio ambiente e uso público.

\section{METODOLOGIA}

O Parque Estadual de Porto Ferreira, criado em 1962 conserva na região nordeste do Estado de São Paulo amostras dos ecossistemas de cerrado, floresta estacional semidecidual e $5 \mathrm{~km}$ de mata ciliar ao longo do rio Mogi-Guaçú.

Para a implantação dos arboretos no PEPF foi adotada a metodologia participativa e colaborativa, principalmente nas semanas comemorativas do Meio Ambiente e da Árvore; onde professores, alunos e representantes da comunidade participavam dos plantios. Nesses eventos, técnicos e monitores da unidade explicavam os passos, desde a coleta de sementes até a manutenção das mudas.

Alguns plantios foram efetuados esporadicamente a pedido da comunidade local e de outras regiões. 
A partir do Plano de Manejo, em 2003 foi adotado o critério de plantio de espécies nativas locais e representantes da Mata Atlântica e do Cerrado do Brasil Central, sem repetir as já estabelecidas, visando aumentar a diversidade da coleção.

\section{O Arboreto Orlando Prezotto}

Esse arboreto foi implantado em 1995 com o objetivo de recuperação de uma área alterada próxima às instalações do Centro de Visitantes. Com a elaboração do Plano de Manejo em 2003, esse arboreto integrou a área de uso público e se localiza na zona de uso intensivo do PEPF (TABANEZ et al., 2003), onde são realizadas atividades de interpretação da natureza, estudo do meio, recreação, observação de pássaros, bem como coleta de sementes e pesquisas científicas.

Em novembro de 2012 durante a comemoração dos 50 anos de criação do Parque, o arboreto foi reorganizado ganhando nova interpretação com a instalação de placas de identificação das espécies e recebendo o nome do Sr. Orlando Prezotto in memória, um conservacionista que na década de 1940 protegia a "Mata do Poção" da Fazenda Santa Mariana, área essa que veio a constituir a unidade de conservação. Essa homenagem foi idealizada pela Pesquisadora Marlene Francisca Tabanez gestora do PEPF por mais de 10 anos e coordenadora do Plano de Manejo.

\section{O Arboreto 50 Anos}

Em 2012 durante uma reunião do Conselho Consultivo para planejamento das comemorações dos 50 anos do PEPF, a Profa. Dra. Maria Ines Pagani do Departamento de Ecologia da UNESP de Rio Claro sugeriu a implantação de um arboreto comemorativo.

Para tanto foram elencadas 50 espécies nativas locais para representar os 50 anos de criação da unidade.

O plantio foi realizado no dia 29.11.2012 com a participação de funcionários do Parque; professores e alunos do ensino fundamental do município, membros do conselho consultivo e, representantes das unidades de conservação da região nordeste do interior do Estado de São Paulo. 
O arboreto localiza-se também na zona de uso intensivo do Parque.

\section{Arboreto dos Ipês}

Esse arboreto foi implantado na perspectiva de melhorar o aspecto paisagístico entre os limites da zona de recuperação de pasto e da zona de uso intensivo - área de uso público, uma vez que a florada da espécie é de beleza cênica.

O plantio foi realizado durante a Semana do Meio Ambiente em junho de 2014 com a participação de professores e alunos do ensino fundamental do município e da empresa ODEBRECHT Ambiental.

\section{A manutenção}

A manutenção dos arboretos é frequente com controle de formigas, retirada das espécies competidoras, coroação e roçada manual das mudas, roçadas mecânicas, retirada de galhos quebrados, podas esporádicas, além da irrigação em períodos de estiagem, na fase inicial do estabelecimento das mudas.

\section{RESULTADOS}

O Arboreto Orlando Prezotto atualmente apresenta 74 espécies pertencentes à 22 famílias botânicas e mais de 100 indivíduos dos ecossistemas de cerrado, floresta e outros associados à Mata Atlântica, à Amazônia, além de algumas exóticas, conforme apresenta a Tabela 1, organizada com base na APG II. 
Tabela 1. Lista das espécies do Arboreto Orlando Prezotto. $\mathrm{CE}=$ Cerrado, $\mathrm{FL}=$ Floresta, $\mathrm{AM}=$ Amazônia e EX= Espécie Exótica.

\begin{tabular}{|c|c|c|}
\hline Família / Espécie & Nome Popular & Vegetação \\
\hline \multicolumn{3}{|l|}{ Anacardiaceae } \\
\hline Myracrodruon urundeuva & Aroeira-preta & FL, CE \\
\hline Spondias mombim & Taperebá & AM \\
\hline Tapirira obtusa & Peito-de-pombo & $\mathrm{FL}, \mathrm{CE}$ \\
\hline \multicolumn{3}{|l|}{ Annonaceae } \\
\hline Annona coriacea & Araticum-do-campo & CE \\
\hline \multicolumn{3}{|l|}{ Apocynaceae } \\
\hline Aspidosperma polyneuron & Peroba-rosa & $\mathrm{FL}$ \\
\hline \multicolumn{3}{|l|}{ Araliaceae } \\
\hline Schefflera morototoni & Mandioqueiro & FL \\
\hline \multicolumn{3}{|l|}{ Bignoniaceae } \\
\hline Handroanthus ochracea & Ipê-amarelo & FL \\
\hline Handroanthus roseo albus & Ipê-branco & $\mathrm{FL}$ \\
\hline Jacaranda cuspidifolia & Caroba & CE \\
\hline Sparattosperma leucanthum & Ipê-cinco-folhas & $\mathrm{FL}$ \\
\hline Tabebuia pentaphylla & Ipê-de-El-Salvador & EX \\
\hline \multicolumn{3}{|l|}{ Boraginaceae } \\
\hline Patagonula americana & Guaiuvira & $\mathrm{FL}$ \\
\hline \multicolumn{3}{|l|}{ Calophyllaceae } \\
\hline Calophyllum brasiliensis & Guanandi & FL, CE \\
\hline \multicolumn{3}{|l|}{ Euphorbiaceae } \\
\hline Alchornea glandulosa & Tapiá & $\mathrm{FL}, \mathrm{CE}$ \\
\hline Croton floribundus & Capixingui & $\mathrm{FL}$ \\
\hline Croton piptocalyx & Caixeta & $\mathrm{FL}$ \\
\hline Mabea fistulifera & Mamona-do-mato & CE \\
\hline Maprounea guianensis & Bonifácio & FL, CE \\
\hline Pachystroma longifolium & Canxim & FL \\
\hline \multicolumn{3}{|l|}{ Fabaceae } \\
\hline Albizia lebbeck & Albízia & EX \\
\hline Albizia niopoides & Farinha-seca & $\mathrm{FL}$ \\
\hline Anadenanthera macrocarpa & Angico & FL \\
\hline Anadenanthera pavonina & Tento-de-mariana & EX \\
\hline Anadenanthera peregrina & Angico-do-morro & $\mathrm{FL}$ \\
\hline Cassia grandis & Acácia & AM \\
\hline Centrolobium tomentosum & Araribá & $\mathrm{FL}$ \\
\hline Copaifera langsdorffi & Óleo-de-copaíba & FL, CE \\
\hline Clitoria fairchildiana & Sombreiro & AM \\
\hline Erythrina crista-galli & Flor-de-coral & $\mathrm{FL}$ \\
\hline Erythrina verna & Suinã & $\mathrm{FL}$ \\
\hline
\end{tabular}


Inga edulis

Leucochloron incuriale

Machaerium stipitatum

Machaerium villosum

Myroxylon peruiferum

Poecilanthe parviflora

Pterogyne nitens

Stryphnodendron polyphyllum

Schizolobium parahyba

Lamiaceae

Vitex cymosa

Lauraceae

Endlicheria paniculata

Nectandra megapotamica

Ocotea porosa

Lecythidaceae

Cariniana legalis

Malvaceae

Luehea divaricata

Pseudobombax grandiflorum

Melastomataceae

Tibouchina granulosa

Meliaceae

Azadirachta indica

Cedrela fissilis

Guarea guidonia

Swietenia macrophylla

Myrtaceae

Campomanesia guazumifolia

Campomanesia phaea

Campomanesia sp.

Eugenia dysenterica

Eugenia brasiliensis

Hexachlamys edulis

Psidium guajava

Syzygium cumini

Phytolaccaceae

Gallesia integrifolia

Rhamnaceae

Colubrina glandulosa

Rhamnidium elaeocarpum
Ingá-de-metro

AM

Angico-rajado

FL

Sapuvinha

$\mathrm{FL}, \mathrm{CE}$

Jacarandá-paulista

FL, CE

Cabreúva

$\mathrm{FL}, \mathrm{CE}$

Coração-de-negro

Amendoim-bravo

Barbatimão-falso

FL, CE

CE

$\mathrm{FL}$

Tarumã

FL

Canela-peluda

FL, CE

Canelinha

$\mathrm{FL}$

Canela-imbuia

FL

Jequitibá-rosa

FL

Açoita-cavalo

FL, CE

Embiruçu

FL

Quaresmeira

CE

$\mathrm{Nim}$

EX

Cedro

FL

Marinheiro

FL

Mogno

AM

Sete-capotes

FL

Cambuci

FL

Gabiroba-de-árvore

FL

Cagaita

CE

Grumixama

FL

Pêssego-do-mato

Goiaba

FL, CE

EX

Jambolão

EX

Pau-d'alho

FL

Saguaraji-vermelho

Saguaraji-amarelo
FL

$\mathrm{FL}$ 


\begin{tabular}{|c|c|c|}
\hline \multicolumn{3}{|l|}{ Rosaceae } \\
\hline Prunus sellowii & Pêssego-bravo & FL \\
\hline \multicolumn{3}{|l|}{ Rubiaceae } \\
\hline Genipa americana & Jenipapo & $\mathrm{FL}$ \\
\hline Tocoyena formosa & Jenipapo-do-cerrado & CE \\
\hline \multicolumn{3}{|l|}{ Rutaceae } \\
\hline Balfourodendron riedelianum & Pau-marfim & $\mathrm{FL}$ \\
\hline Esenbeckia leiocarpa & Guarantã & FL \\
\hline \multicolumn{3}{|l|}{ Salicaceae } \\
\hline Casearia gossypiosperma & Pau-de-espeto & $\mathrm{FL}$ \\
\hline Casearia sylvestris & Guaçatonga & $\mathrm{FL}, \mathrm{CE}$ \\
\hline \multicolumn{3}{|l|}{ Sapindaceae } \\
\hline Dilodendron bipinnatum & Maria-pobre & $\mathrm{FL}, \mathrm{CE}$ \\
\hline \multicolumn{3}{|l|}{ Sapotaceae } \\
\hline Pouteria torta & Guapeva & FL \\
\hline Verbenaceae & & \\
\hline Citharexylum myrianthum & Pau-viola & $\mathrm{FL}$ \\
\hline
\end{tabular}

As flores, os frutos e as sementes da maioria das espécies desse arboreto servem de recurso alimentar para a fauna, atraindo polinizadores e dispersores naturais que contribuem com os processos de regeneração da vegetação.

As espécies Myracrodruon urundeuva e Machaerium villosum encontram-se na lista das ameaçadas de extinção do Estado de São Paulo na categoria vulnerável, enquanto que Aspidosperma polyneuron e Copaifera langsdorffii na categoria quase ameaçada (MAMEDE et al., 2007).

O Arboreto 50 anos conta com 50 indivíduos de 50 espécies pertencentes à 23 famílias botânicas, dos ecossistemas de floresta estacional semidecidual e cerrado, conforme apresenta a Tabela 2, também organizada com base na APG II. 
Tabela 2. Lista das espécies do Arboreto 50 Anos. $\mathrm{CE}=$ Cerrado, FL= Floresta Estacional Semidecidual.

\begin{tabular}{|c|c|c|}
\hline Família / Espécie & Nome Popular & Vegetação \\
\hline \multicolumn{3}{|l|}{ Anacardiaceae } \\
\hline Astronium graveolens & Guaritá & FL \\
\hline Tapirira guianensis & Peito-de-pombo & FL, CE \\
\hline \multicolumn{3}{|l|}{ Annonaceae } \\
\hline Annona cacans & Araticum-cagão & $\mathrm{FL}$ \\
\hline Duguetia lanceolata & Pindaíba & $\mathrm{FL}$ \\
\hline \multicolumn{3}{|l|}{ Apocynaceae } \\
\hline Aspidosperma cylindrocarpon & Peroba-poca & $\mathrm{FL}$ \\
\hline Aspidosperma parvifolium & Guatambu-oliva & FL \\
\hline Aspidosperma polyneuron & Peroba-rosa & FL \\
\hline Aspidosperma ramiflorum & Guatambu-amarelo & FL \\
\hline \multicolumn{3}{|l|}{ Bignoniaceae } \\
\hline Cybistax anthisiphilithica & Ipê-verde & CE \\
\hline Handroanthus ochracea & Ipê-amarelo-do-cerrado & CE \\
\hline Handroanthus vellosoi & Ipê-amarelo-da-mata & $\mathrm{FL}$ \\
\hline \multicolumn{3}{|l|}{ Boraginaceae } \\
\hline Cordia ecaliculata & Café-de-bugre & FL \\
\hline Cordia sellowiana & Juruté & $\mathrm{FL}$ \\
\hline Cordia trichotoma & Louro-pardo & $\mathrm{FL}, \mathrm{CE}$ \\
\hline \multicolumn{3}{|l|}{ Calophyllaceae } \\
\hline Calophyllum brasiliensis & Guanandi & FL \\
\hline \multicolumn{3}{|l|}{ Clusiaceae } \\
\hline Garcinia gardneriana & Bacupari & $\mathrm{FL}$ \\
\hline \multicolumn{3}{|l|}{ Combretaceae } \\
\hline Terminalia argentea & Capitão-do-campo & $\mathrm{FL}, \mathrm{CE}$ \\
\hline \multicolumn{3}{|l|}{ Euphorbiaceae } \\
\hline Mabea fistulifera & Mamoninha & CE \\
\hline Maprounea guianensis & Bonifácio & $\mathrm{FL}$ \\
\hline \multicolumn{3}{|l|}{ Fabaceae } \\
\hline Centrolobium tomentosum & Araribá & $\mathrm{FL}$ \\
\hline Hymenaea courbaril & Jatobá & $\mathrm{FL}, \mathrm{CE}$ \\
\hline Holocalyx balansae & Alecrim-de-campinas & $\mathrm{FL}$ \\
\hline Inga vera & Ingá-do-brejo & $\mathrm{FL}, \mathrm{CE}$ \\
\hline Machaerium villosum & Jacarandá- paulista & $\mathrm{FL}, \mathrm{CE}$ \\
\hline Myroxylon peruiferum & Cabreúva & $\mathrm{FL}, \mathrm{CE}$ \\
\hline Ormosia arborea & Olho-de-cabra & $\mathrm{FL}$ \\
\hline Peltophorum dubium & Canafístula & $\mathrm{FL}$ \\
\hline Platycyamus regnellii & Pau-pereira & $\mathrm{FL}$ \\
\hline \multicolumn{3}{|l|}{ Lauraceae } \\
\hline Criptocarya aschersoniana & Canela & $\mathrm{FL}$ \\
\hline
\end{tabular}


Nectandra megapotamica

Lecythidaceae

Cariniana legalis

Lythraceae

Lafoensia pacari

Malvaceae

Ceiba speciosa

Pseudobombax grandiflorum

Melastomataceae

Tibouchina granulosa

Meliaceae

Cedrela fissilis

Guarea guidonia

Guarea kunthiana

Moraceae

Ficus guaranitica

Maclura tinctoria

Myrtaceae

Campomanesia guazumifolia

Eugenia florida

Rhamnaceae

Rhamnidium elaeocarpum

Rosaceae

Prunus sellowii

Rubiaceae

Alibertia sessilis

Genipa americana

Salicaceae

Casearia sylvestris

Sapindaceae

Cupania vernalis

Diatenopteryx sorbifolia
Canelinha

FL

Jequitibá-rosa

FL

Dedaleiro

FL, CE

Paineira

$\mathrm{FL}$

Embiruçu

FL

Quaresmeira

CE

Cedro-rosa

$\mathrm{FL}$

Marinheiro

$\mathrm{FL}$

Canjambo

$\mathrm{FL}$

Figueira

Taiúva

FL, CE

$\mathrm{FL}$

Sete-capotes

$\mathrm{FL}$

Guamirim

CE

Saguaraji-amarelo

FL

Pêssego-bravo

FL

Marmelo-do-cerrado

CE

Genipapo

FL

Guaçatonga

FL, CE

Camboatã

FL, CE

Maria-preta
$\mathrm{FL}$

A espécie Aspidosperma polyneuron encontra-se na lista das ameaçadas de extinção do Estado de São Paulo na categoria quase ameaçada, enquanto Machaerium villosum e Myroxylon peruiferum na categoria vulnerável (MAMEDE et al., 2007).

No Arboreto dos Ipês foram plantados 18 indivíduos da espécie Handroanthus ochraceus Ipê-amarelo-do-cerrado. 


\section{CONSIDERAÇÕES}

Os arboretos do PEPF foram implantados a partir de 1995 de forma multidisciplinar e participativa e atualmente contam com 94 espécies vegetais, onde a maioria representa os ecossistemas locais e regionais.

No Arboreto Orlando Prezotto são desenvolvidas atividades de educação ambiental, recreação, contemplação da natureza, trabalhos científicos, observação de pássaros, e, por esse motivo, o mesmo se configura em um espaço pedagógico e um atrativo do Parque. A maioria das espécies produzem sementes para a formação de mudas e contribuem com os serviços ecossistêmicos.

Assim, a implantação dos arboretos no PEPF nos últimos 20 anos favoreceu a recuperação de áreas alteradas, o desenvolvimento de processos educativos, a conservação da biodiversidade, os serviços ecossistêmicos, além de serem espaços de vivências e contemplação da natureza, desempenhando funções ambientais, educativas e sociais.

\section{AGRADECIMENTOS}

A todos os funcionários e servidores do PEPF que contribuíram com carinho e zelo com a formação e com a manutenção dos arboretos ao longo dos anos.

\section{REFERÊNCIAS}

BAZARETTI, Viviane Maria et al. Arboreto do Ceplac - espécies arbóreas potenciais ao sistema agrossilvicultural cacaueiro. Unoesc \& Ciência - ACET, Joaçaba, v. 2, n. 1, p. 31-46, jan/jun. 2011.

HERCULIANI, S. et al. Análise das atividades de educação ambiental realizadas no Parque

Estadual da Cantareira. Rev. Inst. Flor., v. 22, n. 1, p. 93-109, 2010.

LORENZI, H. Árvores brasileiras: manual de identificação e cultivo de plantas arbóreas nativas do Brasil. 4a. ed. Nova Odessa, SP: Instituto Plantarum, 2002. vol. 01, 384 p.

MAMEDE, M. C. H. et al. Livro Vermelho das Espécies Vegetais Ameaçadas do Estado de São Paulo. São Paulo: Instituto de Botânica. 2007. 165 p.

SOUZA, Vinicius Castro; LORENZI, Harri. Guia ilustrado para identificação das famílias de Angiospermas da flora brasileira, baseado em APG II. Nova Odessa: Instituto Plantarum, 2008. 704 p.

TABANEZ, Marlene. Francisca et al. Plano de Manejo do Parque Estadual de Porto Ferreira. São Paulo: $\mathrm{O}_{2}$ Estúdio Web, 2003.1CD-ROM. 